\title{
Mutations in the known genes are not the major cause of MED; distinctive phenotypic entities among patients with no identified mutations
}

\author{
Eveliina Jakkula $^{1}$, Outi Mäkitie ${ }^{2,3}$, Malwina Czarny-Ratacjzak ${ }^{4,5}$, Gail C Jackson ${ }^{6,7}$, \\ Rita Damignani ${ }^{2}$, Miki Susic ${ }^{2}$, Michael D Briggs ${ }^{6}$, William G Cole ${ }^{2}$ and \\ Leena Ala-Kokko*,1,4,8
}

\begin{abstract}
${ }^{1}$ Collagen Research Unit, Biocenter and Department of Medical Biochemistry and Molecular Biology, University of Oulu, Oulu, Finland; ${ }^{2}$ Hospital for Sick Children, Division of Orthopaedics, Toronto, Ontario, Canada; ${ }^{3}$ Hospital for Children and Adolescents, Helsinki University Hospital, Helsinki, Finland; ${ }^{4}$ Center for Gene Therapy, New Orleans, USA;

${ }^{5}$ Department of Medical Genetics, Karol Marcinkowski University of Medical Sciences, Poznan, Poland; ${ }^{6}$ Wellcome Trust Centre for Cell Matrix Research, School of Biological Sciences, University of Manchester, UK; ${ }^{7}$ National Genetics Reference Laboratory (Manchester), Regional Genetics Service, St Mary's Hospital, Manchester, UK; ${ }^{8}$ Department of Medicine, Tulane University Health Sciences Center, New Orleans, USA
\end{abstract}

Multiple epiphyseal dysplasia (MED) is a clinically and genetically heterogeneous chondrodysplasia. Mutations in six genes (COMP, COL9A1, COL9A2, COL9A3, MATN3 and DTDST) have been reported, but the genotype-phenotype correlations and the proportions of cases due to mutations in these genes are still not well characterized. We performed a clinical, radiological and molecular analysis of known MED genes on 29 consecutive MED patients. The mutation analysis resulted in identification of the DTDST mutation in four patients (14\%), the COMP mutation in three (10\%) and the MATN3 mutation in three (10\%). Thus, a disease-causing mutation was identified in 10 patients altogether (34\%). The phenotypic features observed in the patients with mutations were in accordance with previously described phenotypes, but two new distinct phenotypic entities were identified in patients in whom no mutation was found. One of them was characterized by severe, early-onset dysplasia of the proximal femurs with almost complete absence of the secondary ossification centres and abnormal development of the femoral necks. The other phenotype was characterized by 'mini-epiphyses', resulting in severe dysplasia of the proximal femoral heads. The findings suggest that mutations in the known genes are not the major cause of MED and are responsible for less than half of the cases. The existence of additional MED loci is supported by the exclusion of known loci by mutation analysis and finding of specific subgroups among these patients. European Journal of Human Genetics (2005) 13, 292-301. doi:10.1038/sj.ejhg.5201314

Published online 3 November 2004

Keywords: MED; chondrodysplasia; COMP; DTDST; MATN3; COL9A1; COL9A2; COL9A2; cartilage

\footnotetext{
${ }^{*}$ Correspondence: Dr L Ala-Kokko, Department of Medical Biochemistry and Molecular Biology, Collagen Research Unit, University of Oulu, P.O Box 5000, 90014 Oulu, Finland. Tel: + 35885375751 ; Fax: + 3588537 5811; E-mail: leena.ala-kokko@oulu.fi Received 17 June 2004; revised 8 September 2004; accepted 8 September 2004
}

Introduction

Multiple epiphyseal dysplasia (MED) is a heterogeneous chondrodysplasia with autosomal dominant or recessive inheritance characterized by joint pain and stiffness, waddling gait and/or mild short stature in childhood. Radiographic findings include delayed and 
irregular ossification of the epiphyses in multiple joints. Spinal changes, if present, are mild. ${ }^{1-3}$

The disorder is also genetically heterogeneous. Mutations in the cartilage oligomeric matrix protein (COMP) gene cause a dominantly inherited form of MED (EDM1, MIM 600310). COMP is a $550 \mathrm{kDa}$ pentameric glycoprotein of the extracellular matrix (ECM) and is found predominantly in cartilage, tendon and ligament. ${ }^{2}$ In all, 22 COMP mutations have been identified in 27 MED patients to date. $^{2,4-6}$ Mutations in COMP also cause pseudoachondroplasia (PSACH, MIM 177170), which is a more severe chondrodysplasia, but the EDM1 and PSACH phenotypes partly overlap. ${ }^{2}$

Mutations in the genes coding for the collagen IX $\alpha$ chains have been shown to cause dominantly inherited MED, and altogether eight such mutations have been identified in nine MED families. ${ }^{2,7}$ All these mutations cluster in the splice-donor or acceptor site of exon 3 of COL9A2 (EDM2, MIM 600204) or COL9A3 (EDM3, MIM $600969)$ or in the splice-donor site of exon 8 of COL9A1.

The fifth gene identified as causing dominantly inherited MED (EDM5, MIM 602019) is MATN $3,{ }^{8}$ which belongs to the matrilin protein family, the members of which are multidomain oligomeric ECM proteins expressed in cartilage and bone. Seven missense mutations in MATN3 have been identified in 10 MED families to date, one of them, $\mathrm{R}^{121} \mathrm{~W}$, being recurrent. All these mutations are clustered in exon 2, which encodes the vWFA-like domain of MATN3. ${ }^{8-10}$ Recently, an association of MATN3 $\mathrm{Thr}^{303} \mathrm{Met}$ polymorphism with hand OA was reported. ${ }^{11}$

Patients with the recessive form of MED (rMED) (EDM4, MIM 226900) are either homozygous or compound heterozygous for mutations in the gene for the sulphate transporter, DTDST (SLC26A2). ${ }^{12-15}$ Mutations in this gene can also cause more severe recessive disorders such as achondrogenesis 1B (AGC1B, MIM 600972), atelosteogenesis type 2 (AO2, MIM 256050) and diastrophic dysplasia (DTD, MIM 222600). ${ }^{16}$ The homozygous $\mathrm{R}^{279} \mathrm{~W}$ mutation is the most common mutation in patients with rMED. ${ }^{14}$

MED patients with COMP mutations usually have the most severe changes in the hip joints and a progressive disease leading to early-onset hip OA, requiring joint replacement. In contrast, patients with collagen IX mutations typically have relative sparing of the hip joints, while the most drastic radiographic findings are observed in the knees. ${ }^{2,17}$ The hips and knees are the most commonly affected joints in patients with MATN3 mutations, but the hip involvement is intermediate relative to that caused by COMP or collagen IX mutations. ${ }^{8}$ The presence of epiphyseal changes in combination with a double-layered patella and/or clubfoot is characteristic of rMED caused by DTDST mutations. ${ }^{12,14-16}$

We report here on genetic, clinical and radiographic findings in a cohort of MED patients followed at a paediatric orthopaedic clinic in Toronto, Canada. The findings suggest that mutations in the known genes are not the major cause of MED and are responsible for less than half of the cases. Clinical and radiographic assessment of the patients with no identified mutations in the known loci revealed distinctive phenotypic subgroups, suggesting that additional, as yet uncharacterised, loci are likely to exist.

\section{Patients and methods \\ Patients}

Patients followed (by WG Cole) at the Orthopaedic Clinic, The Hospital for Sick Children, Toronto, during 1996-2002 for MED were eligible for inclusion in the cohort. The diagnosis of MED was based on typical clinical and radiographic features. Altogether 30 consecutive unrelated patients with MED were included. Detailed clinical and mutation data have been reported previously for four of these patients. Three of these patients with clinical findings consistent with rMED had homozygous DTDST mutations; ${ }^{14,15}$ one had radiographic findings typical of EDM1 and was found to have a COMP mutation. ${ }^{18}$ Of the remaining 26 patients, 25 consented to participate in the study. Their hospital records were reviewed for symptoms, clinical features and family history, and radiographs were assessed for skeletal characteristics of MED. The study was approved by the local Research Ethics Boards and signed informed consent was obtained from the subjects or their parents.

Genomic DNA was isolated by standard methods from peripheral blood lymphocytes obtained from the patients (in 20 cases) or from an affected parent (in five cases; patients 3, 7, 9, 13 and 17, Table 1). DNA was analysed for the presence of disease-causing mutations and other sequence variations in all the genes known to be associated with MED (COMP, MATN3, COL9A1, COL9A2, COL9A3 and DTDST). If a mutation was identified in a patient, DNA samples were also obtained from the parents and sibs and their clinical data were reviewed if available. If the initial mutation screening was negative and there were affected family members in at least three generations, a linkage approach was used to confirm the findings (patients 7 and 9). In addition, DNA samples from 100 unaffected, unrelated controls were used to determine whether the novel sequence variations identified were likely to be common polymorphisms or disease-causing mutations.

\section{Mutation analysis of COL9A1, COL9A2, COL9A3, COMP, DTDST and MATN3}

Since all collagen IX gene mutations are clustered in the splice sites of exon 3 of COL9A2 or COL9A3 or the splice donor site of exon 8 of COL9A1, ${ }^{2}$ exons 8-10 of COL9A1, exons 2-4 of COL9A2 and COL9A3 and the flanking sequences were amplified by PCR to 
Table 1 Clinical, radiological and genetic findings in the MED patients

Radiographic findings

$\begin{array}{ccccccccccc}\begin{array}{l}\text { Patient } \\ \#\end{array} & \begin{array}{c}\text { Age } \\ \text { (years) }\end{array} & \text { Sex } & \begin{array}{c}\text { Family } \\ \text { history }\end{array} & \begin{array}{c}\text { Age at } \\ \text { onset } \\ \text { (years) }\end{array} & \begin{array}{c}\text { Height } \\ \text { percentile (\%) }\end{array} & \text { Surgery } & \text { Hips } & \text { Knees } & \text { Spine } & \text { Other joints } \\ \text { Mutation }\end{array}$

$\mathrm{M}=$ male, $\mathrm{F}=$ female, $\mathrm{OT}=$ osteotomy, $\mathrm{NA}=$ not available, $\mathrm{F}=$ feet, $\mathrm{H}=$ hands, $\mathrm{S}=$ shoulders, $\mathrm{OCD}=$ osteochondritis dissecans, $\mathrm{A}=$ ankles, $\mathrm{THR}=$ total hip replacement.

Table 2 PCR primers and conditions for the analysis of the DTDST gene

\begin{tabular}{|c|c|c|c|c|c|}
\hline Exon & $P C R$ primers & Primer sequences & Size $(b p)^{a}$ & $\operatorname{Tm}^{\mathrm{b}}\left({ }^{\circ} \mathrm{C}\right)$ & Sequencing primer \\
\hline e1 & $\begin{array}{l}\text { dtd1f, } \\
\text { dtd1r }\end{array}$ & $\begin{array}{l}\text { 5'- AGC ACT TCA AAA CTC TGG GC } \\
\text { 5'- GAC CCC TGA TCT GGG ATT CT }\end{array}$ & 639 & 58 & dtd1f \\
\hline e2 & $\begin{array}{l}\text { dtd2f } \\
\text { dtd } 2 r\end{array}$ & $\begin{array}{l}\text { 5'- AGG AAG CTG AAC CAT CTA TCT C } \\
5^{\prime}-\text { CCT TCA TAT ATC CTG AAC TCT C }\end{array}$ & 857 & 60 & $\begin{array}{l}\text { dtdt2f, } \\
\text { dtd } 2 r\end{array}$ \\
\hline $\begin{array}{l}\text { e3 } \\
\left(5^{\prime}\right)\end{array}$ & $\begin{array}{l}\text { dtd3f } \\
\text { dtd3rs1 }\end{array}$ & $\begin{array}{l}\text { 5'- TAG CTC TGA CAT TCT GTG AT } \\
5^{\prime}-\text { GAA TTG CAC TGC AGT CAA TCA C }\end{array}$ & 1389 & 60 & dtd3f \\
\hline $\begin{array}{l}\mathrm{e} 3 \\
\left(3^{\prime}\right)\end{array}$ & $\begin{array}{l}\text { dtd3fs1 } \\
\text { dtd3r }\end{array}$ & $\begin{array}{l}\text { 5'- GCT GGA CAT ATT CCC ACT G } \\
\text { 5'- CAT TCC AAG AAT ACT TCA TAT CTG }\end{array}$ & 1386 & 60 & $\begin{array}{l}\text { dtd3fs } 1 \\
\text { dtd3r }\end{array}$ \\
\hline
\end{tabular}

${ }^{\mathrm{a}} \mathrm{bp}=$ base pair.

${ }^{\mathrm{b}} T_{\mathrm{m}}=$ annealing temperature.

obtain products of $252-396$ bp for mutation screening by conformation-sensitive gel electrophoresis (CSGE). ${ }^{6}$ All samples were also sequenced using an ABI PRISM ${ }^{\mathrm{TM}} 377$ or 3100 Sequencer and BigDye Terminator Cycle Sequencing Kit (Applied Biosystems).

Sequences corresponding to exons 1-7 of COMP and the flanking sequences were amplified by PCR and analysed by CSGE. Samples that contained heteroduplexes in CSGE analysis were sequenced to identify the underlying sequence variation. As all COMP mutations have been reported to be located between exons 8 and $19,{ }^{2}$ these exons and the flanking intronic sequences were analysed by both CSGE and sequencing. ${ }^{6}$
The DTDST gene exons (1-3) and their flanking sequences were amplified by PCR in four fragments, which were analysed by sequencing. The primer sequences, sizes of the PCR products and primers used in sequencing are shown in Table 2, and the details of PCR amplifications are described in the on-line supplement.

Mutation analysis of all the exons and flanking the sequences of MATN3 had been performed previously in 20 of the 25 patients and the results of this analysis reported earlier. ${ }^{10,19}$ As five additional patients had been ascertained since the initial analysis, MATN3 exon 2 was amplified from their samples as previously described ${ }^{10}$ and analysed for sequence variations by CSGE and sequencing. 


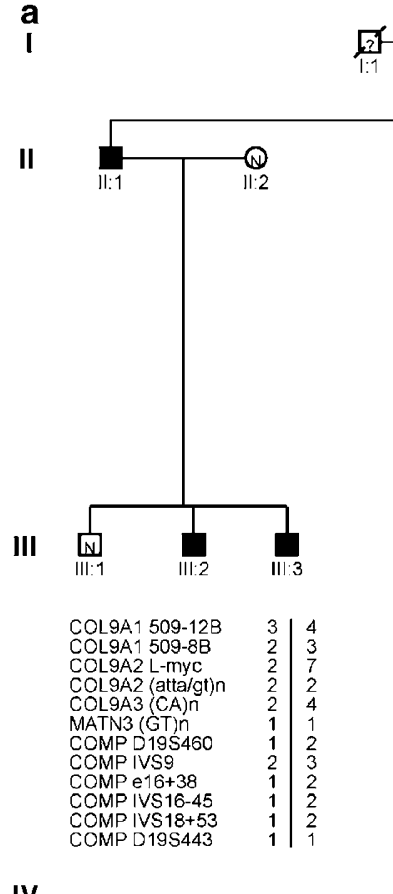

IV

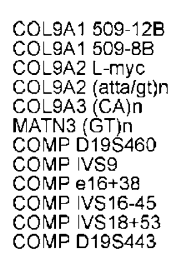

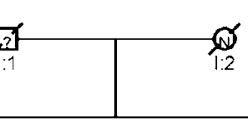
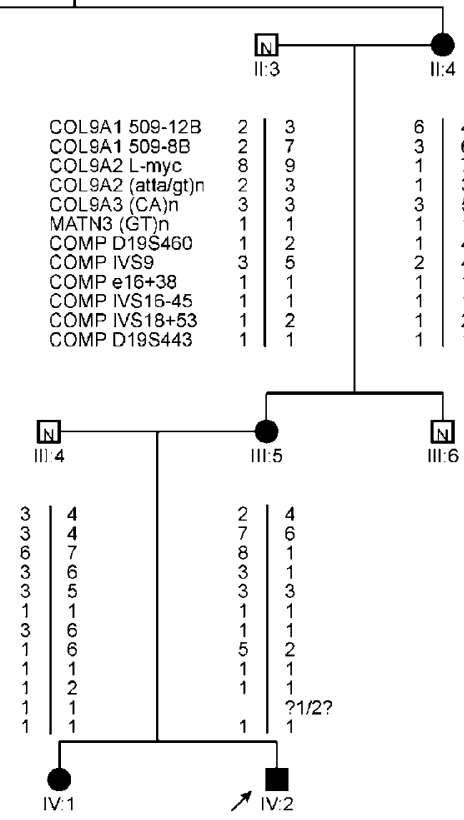

\begin{tabular}{l|l}
4 & 4 \\
3 & 6 \\
6 & 8 \\
6 & 3 \\
3 & 3 \\
1 & 1 \\
6 & 1 \\
6 & 5 \\
1 & 1 \\
1 & 1 \\
1 & 1 \\
1 & 1
\end{tabular}

b

I

॥

III

III

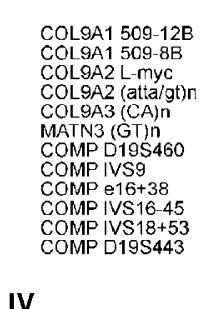

IV

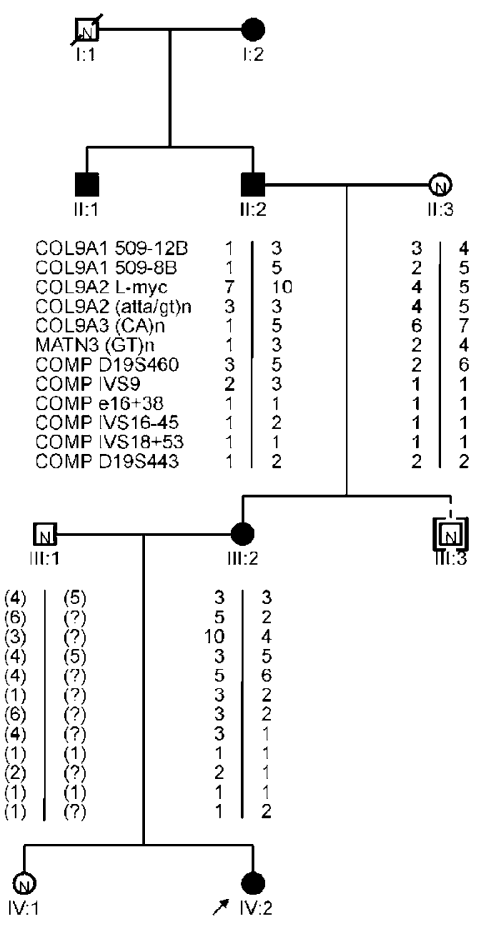

COL9A1 509-12B COLAA $509-8 B$
COL9A2 L-MYC COL9A2 L-myc
COL9A2 (atta/gt) COL9A2 (attalgt)
COLA3 (CA)n MATN3 (GT) n COMPIVS9 COMP $16+38$ COMP IVS $16-45$ COMP IVS $18+53$
COMP D19S443 \begin{tabular}{l|l}
5 & 3 \\
6 & 5 \\
3 & 4 \\
4 & 3 \\
4 & 6 \\
1 & 3 \\
6 & 3 \\
4 & 3 \\
1 & 1 \\
2 & 2 \\
1 & 1 \\
1 & 1
\end{tabular}

Figure 1 Family pedigrees and results of the genotyping of known MED genes. (a) Family of patient 7, (b) family of patient 9. Black circles (females) and squares (males) indicate MED individuals. The probands are denoted by diagonal arrows. The alleles for each marker are shown below the symbols. The bar between alleles separates different haplotypes. The question marks besides alleles indicate that it was not possible to deduce unambiguously which allele came from which parent.

\section{Genotyping of families}

The known loci for autosomal dominant MED (COL9A1, COL9A2, COL9A3, COMP and MATN3) were analysed for linkage in the families of patients 7 and 9 (Figure $1 \mathrm{a}$ and $\mathrm{b}$ ). Markers 509-8B2 (in intron 5 of COL9A1) and 509-12B1 ( $<15 \mathrm{~kb} 5^{\prime}$ of COL9A1) were used for COL9A1, the ATTA/ GT repeat $\left(<29 \mathrm{~kb} 5^{\prime}\right.$ of COL9A2) and L-myc $(200 \mathrm{~kb} \mathrm{5'}$ of COL9A2) for COL9A2, the CA repeat (16 kb $5^{\prime}$ of COL9A3) for COL9A3, D19S460 (450 kb $5^{\prime}$ of COMP), the TAAA repeat (in intron 9 of COMP) and D19S443 (225 kb $3^{\prime}$ of COMP) for COMP and the GT repeat (in intron 3 of MATN3) for $M A T N .^{2,20}$ In addition, three COMP polymorphisms, c. $1755 \mathrm{G}>\mathrm{A}$, c.1915-45C $>\mathrm{T}$ and c.2228-40T $>\mathrm{C}$, were genotyped in both affected families by sequencing.

\section{RNA analysis}

Total RNA from EBV-transformed lymphoblasts of patients 7 and 9, respectively, was isolated with the RNeasy kit (Qiagen) and used as a template for RT-PCR analysis of
COMP. The locations of the primers used in the PCR analysis and the sizes of the PCR products are shown in Figure 2 and details of RT-PCR analysis are described in the on-line supplement.

\section{Results COMP}

The analysis of COMP identified two novel mutations (Table 3). Patient 20 had a c.1265A to C transversion, which resulted in the substitution of Ala for the conserved Asp422 in the sixth calcium-binding thrombospondin type III (T3) repeat. This variation was not observed in any of the controls $(n=100)$. The proband had a history of knee pain and gait abnormality since the age of 8 years. At the age of 17 years, she had short stature $(143 \mathrm{~cm}$, «3rd percentile), generalized joint laxity and genua valga. Radiologically she had relatively mild changes consistent with EDM1: the femoral heads were small and irregular and 


\begin{tabular}{|c|c|c|c|c|c|c|c|c|c|c|c|c|c|c|c|c|c|c|}
\hline 1 & 2 & 3 & 4 & 5 & 6 & 7 & 8 & 9 & 10 & $\begin{array}{ll}11 & 12\end{array}$ & 13 & 14 & 15 & 16 & 17 & 18 & 19 & 3'-UTR \\
\hline \multirow[t]{3}{*}{$\stackrel{\text { E1F }}{\Longrightarrow}$} & & & & & & & & $\stackrel{\operatorname{cc} 2 \mathrm{~F}}{\rightrightarrows}$ & & $525 \mathrm{bp}$ & & & & & & & & \\
\hline & & & & & & & & & $873 \mathrm{~b}$ & & & $\mathrm{E} 14 \mathrm{R}$ & & & & & & \\
\hline & & & & & & & & $506 \mathrm{bp}$ & & $\stackrel{c c 1 R}{\longleftarrow}$ & & $\stackrel{\operatorname{cc} 6 F}{\longrightarrow}$ & & & & & & $\stackrel{\operatorname{cc} 6 R}{\longleftarrow}$ \\
\hline
\end{tabular}

Figure 2 Primers used for RT-PCR analysis of COMP. COMP CDNA, with exons marked as boxes, is drawn to scale. The locations of the primers are indicated by arrows above each line corresponding to a fragment amplified by PCR. The sizes of the fragments are shown above them.

Table 3 Sequence variations in COL9A1, COL9A2, COL9A3, COMP and DTDST observed in the MED patients

\begin{tabular}{|c|c|c|c|c|}
\hline Gene & Location & Variation & Frequency in MED patients ${ }^{\mathrm{a}}$ & Reference \\
\hline \multirow[t]{12}{*}{ COMP } & E1 ( $5^{\prime}$ UTR) & c. $-9 \mathrm{G}>\mathrm{T}$ & $1 / 26$ & \multirow{12}{*}{$\begin{array}{l}\text { Mabuchi et al }(2001)^{25} \\
\text { Briggs et al }(2002)^{2}\end{array}$} \\
\hline & IVS1 & c. $79-93 \mathrm{~g}>\mathrm{c}^{\mathrm{b}}$ & $1 / 26$ & \\
\hline & IVS2 & c. $165+41 \mathrm{~g}>\mathrm{c}$ & $5 / 26$ & \\
\hline & IVS3 & c. $218-14 c>t$ & $1 / 26$ & \\
\hline & IVS5 & c. $528+76 \mathrm{t}>\mathrm{c}$ & $1 / 26$ & \\
\hline & E11 & c. $1156 \mathrm{~A}>\mathrm{G} ; \mathrm{Asn}^{386} \mathrm{Asp}$ & $1 / 26$ & \\
\hline & IVS11 & c. $1255-5 c>t^{b}$ & $1 / 26$ & \\
\hline & E12 & C. $1265 \mathrm{~A}>\mathrm{C} ; \mathrm{Asp}_{585}^{422} \mathrm{Ala}$ & $1 / 26$ & \\
\hline & E16 & c. $1755 \mathrm{G}>\mathrm{A} ; \mathrm{Thr}^{585} \mathrm{Thr}$ & $3 / 26$ & \\
\hline & IVS16 & c. $1915-45 c>t$ & $11 / 26$ & \\
\hline & E18 & c. $2204 \mathrm{C}>\mathrm{T} ; \mathrm{Ala}^{735} \mathrm{Val}$ & $1 / 26$ & \\
\hline & IVS18 & c. $2228-40 t>c$ & $7 / 26$ & \\
\hline \multirow[t]{3}{*}{ COL9A1 } & IVS8 & $c .876+13 c>t$ & $1 / 26$ & Jakkula et al (2003) \\
\hline & IVS8 & $c .877-23 c>g$ & $3 / 26$ & Loughlin et al (2002) ${ }^{26}$ \\
\hline & IVS10 & c. $975+45 g>a$ & $14 / 26$ & Loughlin et al (2002) $^{26}$ \\
\hline \multirow{3}{*}{$\begin{array}{l}\text { COL9A2 } \\
\text { COL9A3 }\end{array}$} & IVS4 & c. $249+36 c>a$ & $1 / 26$ & Jakkula et al (2003) \\
\hline & & c. $93 \mathrm{C}>\mathrm{A} ;$ Pro $^{29}$ Pro & $6 / 26$ & Jakkula et al (2003) ${ }^{6}$ \\
\hline & & c. $129 \mathrm{C}>\mathrm{T}$; Pro ${ }^{43}$ Pro & $6 / 26$ & \\
\hline \multirow[t]{6}{*}{ DTDST } & 5 flanking region & g. $10503026 \mathrm{G}>\mathrm{T}$ & $9 / 26$ & \multirow{6}{*}{$\begin{array}{l}\text { Rossi et al }(2001)^{16} \text { (as homoz in DTD) } \\
\text { Hästbacka }(1996)^{27} \text {, Superti-Furga } \\
\text { et al (1999) } \\
\text { Superti-Furga et al }(1999)^{12} \\
\text { Rossi et al }(2001)^{16} \text { (as homoz in DTD) }^{28} \\
\text { Hästbacka }(1994)^{28}\end{array}$} \\
\hline & $\begin{array}{l}\text { E1 (5'UTR) } \\
\text { E3 }\end{array}$ & $\begin{array}{l}\text { g. } 10503322 \mathrm{G}>\mathrm{C} \\
\text { c. } 229 \mathrm{~A}>\mathrm{C}, \mathrm{Asn}^{77} \mathrm{His}^{\mathrm{c}}\end{array}$ & $\begin{array}{l}1 / 26 \\
1 / 26\end{array}$ & \\
\hline & E3 & c. $832 \mathrm{C}>\mathrm{T} ; \mathrm{Arg}^{279} \operatorname{Trp}$ (hetero) & $1 / 26$ & \\
\hline & $\begin{array}{l}\text { E3 } \\
\text { E3 }\end{array}$ & $\begin{array}{l}\mathrm{c.832C}>\mathrm{T} ; \mathrm{Arg}^{279} \operatorname{Trp} \text { (homoz) } \\
\text { c. } 1410 \mathrm{~A}>\mathrm{G}, \mathrm{Val}^{470} \mathrm{Val}^{\mathrm{C}}\end{array}$ & $\begin{array}{l}1 / 26 \\
1 / 26\end{array}$ & \\
\hline & E3 & c. $1474 \mathrm{C}>\mathrm{T}, \mathrm{Arg}^{492} \mathrm{Trp}^{\mathrm{d}}$ & $1 / 26$ & \\
\hline & E3 & c. $2065 \mathrm{~A}>\mathrm{T}$, $\mathrm{Thr}^{689} \mathrm{Ser}$ & $6 / 26$ & \\
\hline
\end{tabular}

The disease-causing mutations identified here are in bold. Homoz = homozygous, Hetero= heterozygous.

${ }^{a}$ The analysis was performed on samples from 20 patients and on a sample from an affected parent in 5 cases. In addition, the sample from an affected uncle of patient 7 was included in the analysis.

b Patient 1 has an $\operatorname{Arg}^{121}$ Trp mutation in MATN3. COMP changes are likely to be neutral.

${ }^{C}$ Patient 20 has an $\mathrm{Asp}^{422}$ Ala mutation in COMP. DTDST changes are likely to be neutral.

dPatient 13 has a $11 e^{192}$ Asn mutation in MATN3. DTDST changes are likely to be neutral.

Genebank accession numbers: COMP NM_000095.2 (cDNA); COL9A1 NM_001851.2 (cDNA, long form); COL9A2 NM_001852.3 (cDNA); COL9A3 NM_001853.2 (cDNA); DTDST (SLC26A2) NM_000112.2 (cDNA), NT_029289.10 (gDNA).

the femoral necks were short and broad. The spine and hands were normal. Her parents were unaffected; neither of them had the same sequence variation, suggesting a de novo mutation.

Patient 10 was found to have c. $2004 \mathrm{C}>\mathrm{T}$ transition in exon 18 predicted to cause $\mathrm{Ala}^{735}$ Val substitution in the Cterminal domain of COMP. He had had limited mobility of the hips since the age of 6 months and abnormal gait and knee and hip pain since the age of 4 years. At 6 years, his height was $117 \mathrm{~cm}$ (between the 50th and 75th percen- tiles). He had difficulty in walking, mild proximal muscular hypotonia in the lower extremities and hyperactive deep tendon reflexes. Neuromuscular assessment, including muscle biopsy and electrophysiological investigations, was normal, but movement of the hips was limited. Radiographic findings included small, deformed proximal femoral epiphyses, broad femoral necks and irregular acetabuli. His mother had a history of knee and hip pain since her teens and bilateral THR in adulthood. The proband had inherited the mutation from his mother. 
None of the controls $(n=100)$ had this variation, and comparison of amino-acid sequences between species showed that the amino acid was highly conserved (data not shown).

\section{DTDST}

Analysis of DTDST led to the identification of seven sequence variations, usually in a heterozygous state (Table 3). One of the patients, 19, was found to be homozygous for the most common rMED mutation, $\operatorname{Arg}^{279}$ Trp. His clinical history was typical of rMED, consisting of clubfoot deformity, short stature $(138 \mathrm{~cm}$ at 14 years; $\ll 3$ rd percentile) and knee pain. His radiographs showed generalized epiphyseal dysplasia, especially in the proximal femurs, short, broad metacarpals and mild flattening and irregularity of the vertebrae; no lateral knee films were available for assessment of the patella. Both of his unaffected parents were heterozygous carriers of the mutation.

\section{MATN3}

Two mutations in MATN3 were identified, $\operatorname{Arg}{ }^{121} \operatorname{Trp}$ in patient 1 and Ile ${ }^{192}$ Asn in patients 4 and 13 (Table 1). The clinical and radiographic features of patients 1 and 13 have been published separately. ${ }^{10,19}$ Patient 4 had a positive family history of MED, with several affected family members. He became symptomatic at age 8 years, with knee and hip pain. At 11 years his height was $140 \mathrm{~cm}$ (between the 25th and 50th percentiles), he walked with a limp and he had limited hip rotations. Innominate osteotomy was performed at 11 years (Table 1 ). The radiographic findings were very similar to those described in EDM5: small, flat, irregular proximal femoral epiphyses and small distal femoral and proximal tibial epiphyses with irregular contours associated with mild metaphyseal irregularity. The same mutation was identified in his affected sister, who had been asymptomatic until the age of 9 years despite bilateral dysplasia of the femoral epihyses and coxa vara.

\section{COL9A1, COL9A2 and COL9A3}

CSGE and sequencing analysis of exons 8-10 of COL9A1 and exons 2-4 of COL9A2 and COL9A3 did not reveal any disease-causing mutations.

Linkage analysis of candidate genes in the families of patients 7 and 9 and RNA analysis of COMP Analyses of samples from the affected mother (Figure 1a, III:5) and the affected cousin of her mother (Figure 1a, III:3) of patient 7 (Figure 1a) and the affected mother of patient 9 (Figure 1b) failed to identify any disease-causing mutations. In both families, the phenotype was typical of MED and the family history suggested autosomal dominant inheritance. Clinical data and blood samples were therefore obtained from family members (Figure $1 \mathrm{a}$ and $\mathrm{b}$ ) and linkage analysis with markers for COL9A1, COL9A2,
COL9A3, COMP and MATN3 was performed. Recombinations were initially observed between COL9A1, COL9A2 and $C O L 9 A 3$ and the MED phenotype in family 7 (Figure 1a). At that point, the status of the sister of patient 7 (IV:1, Figure 1a) was uncertain and RNA analysis of COMP was started. Later she was found to have clinical and radiographic findings consistent with MED, resulting in a recombination of COMP with the phenotype (Figure 1a). The loci for MATN3 could not be excluded in family 7 . Recombinations between COL9A2, COL9A3 and MATN3 and the phenotype were observed in family 9 (Figure 1b), the loci for COL9A1 and COMP could not be excluded.

RNA analysis of COMP in patients 7 and 9 was performed to search for possible mutations, resulting in larger gene rearrangements. Expression of both COMP alleles could not be verified, since the exonic polymorphisms reported to date (c.279C $>$ A, Pro ${ }^{93}$ Pro; c. $468 G>$ T, Pro ${ }^{156}$ Pro; c. $1156 \mathrm{~A}>\mathrm{G}, \mathrm{Asn}^{386} \mathrm{Asp}$ and c. $1755 \mathrm{G}>\mathrm{A}$; $\mathrm{Thr}^{585} \mathrm{Thr}$ ) are rare and were not present in these patients. No size differences or sequence variations were detected in the cDNA fragments (data not shown). Thus, the negative results of mutation analysis at the DNA and cDNA levels and the recombination observed in linkage analysis with COMP confirm that the disease-causing gene in family 7 is not COMP.

Radiographic findings in patients without mutations In all, 18 of the 19 patients without a mutation had hip involvement, and in 15 of them it was regarded as severe. The radiographic findings in the hips were very variable and comprised delayed appearance, small size, irregular contour and/or fragmentation of the proximal femoral epiphyses, irregular acetabuli, a wide and/or short femoral neck and/or coxa vara (Figures 3 and 4). These changes were always bilateral, but the degree of severity varied between the left and right hip in some cases. The patient with no hip involvement (18) had changes consistent with osteochondritis dissecans affecting the knees and ankles bilaterally.

Knee radiographs were available for 16 of the 19 patients, and all except one had radiographic changes in the knees (Table 1). These were usually much milder than the changes in the hips, however, and were characterized by small, irregular, flat and/or asymmetric epiphyses. None of the 11 patients with a lateral knee radiograph had a double-layered patella, but a small patella with irregular contour was observed in five patients. Hand radiographs, available for 12 patients, were usually normal. Spinal radiographs were available for 20 patients and mild spinal changes were seen in seven of them. Ankle radiographs, available for 17 patients, showed epiphyseal changes in seven cases (Table 1 ).

New phenotypic entities Two distinctive new phenotypic entities were identified among the patients with no 


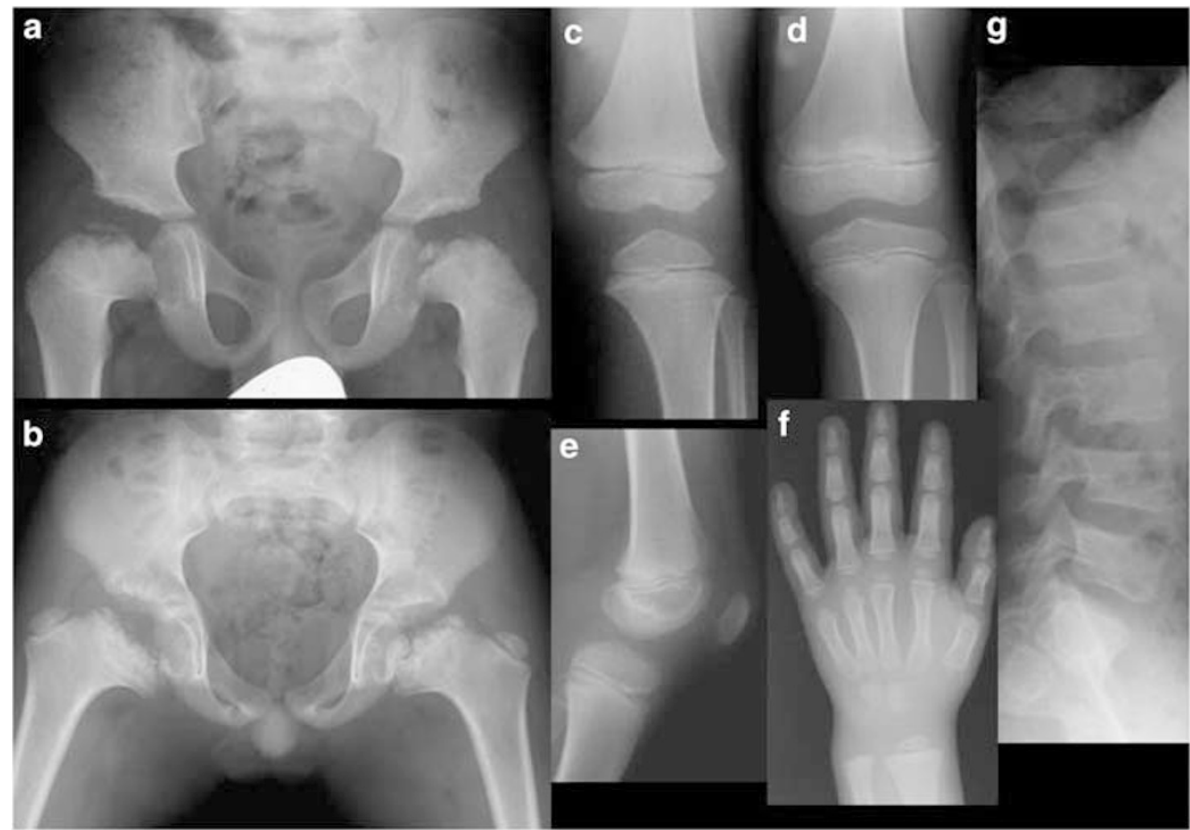

Figure 3 Patient 25 had bilateral hip and knee pain since age 2.5 years. At age 4 years he walked with a limp and had a mild genua valga deformity and limited hip rotations. The clinical symptoms progressed with age. Anterior-posterior (AP) radiographs of the pelvis ((a) at 4 years and (b) at 8 years) showed severe hip involvement with marked deformity or absence of the proximal femoral epiphyses. The femoral necks were wide and short and in varus position. Knee radiographs (AP) ((c) at 4 years and (d) at 8 years) showed mild asymmetry of the distal femoral epiphysis and very mild flaring and irregularity of the distal femoral and proximal tibial metaphyses. Lateral view of the knee, ((e) at 8 years) showed normal patella. Hand radiograph $((\mathbf{f})$ at 4 years) was normal. Lateral view of the spine $((\mathbf{g})$ at 8 years $)$ showed mild irregularity and flattening of the vertebral bodies.

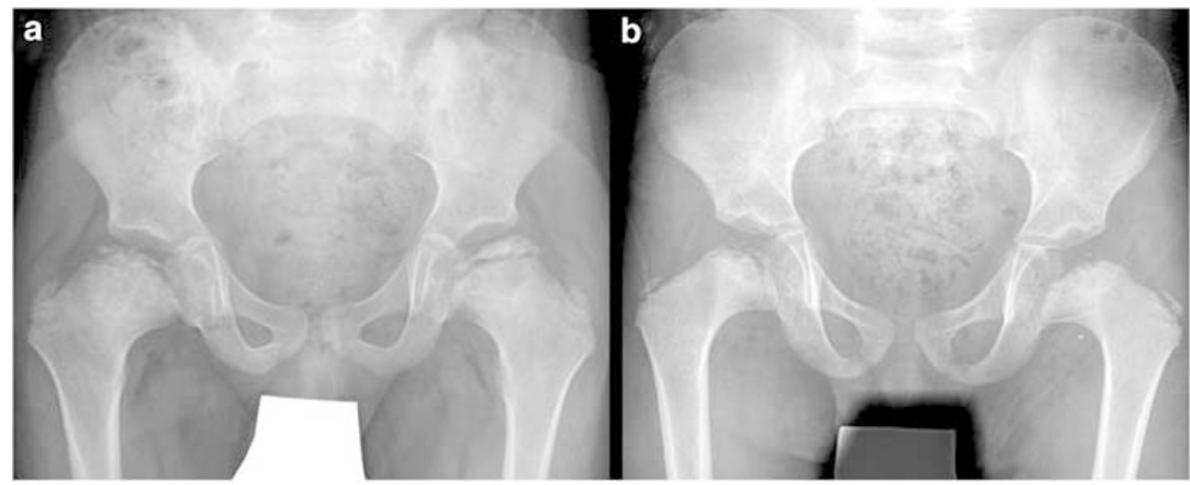

Figure 4 AP radiographs of the pelvis in patient 24 ((a) at 5 years) and in patient 17 ((b) at 5 years) showed similar severe hip involvement with marked deformity or absence of the proximal femoral epiphyses as seen in patient 25 (see Figure 3 ). The femoral necks were short, wide and in varus position.

observed mutations. Three of them (patients 17, 24 and 25, Table 1) shared very similar clinical and radiographic features, having presented with hip or knee symptoms in early childhood. One of them (patient 17) had a positive family history consistent with autosomal dominant inheritance: his affected mother had a history of bilateral total hip replacement and his affected maternal grandmother a history of bilateral hip and knee replacements.
None of the three patients had undergone surgery. Their radiographic findings at age 5-8 years showed very severe hip involvement with marked deformity or complete absence of the proximal femoral secondary ossification centres (Figures 3 and 4). The femoral necks were wide and short, with irregular metaphyses. All three cases had coxa vara. The distal femoral epiphyses were slightly flat and asymmetric, while the metaphyses of the distal femurs and 
the distal tibias showed mild flaring and irregularity. Two of the three had mild spinal changes with reduced vertebral height (Figure 3). Other joints showed mild or no changes.

Patient 16 had unique radiographic findings with strikingly small secondary ossification centres (mini-epiphyses) in all joints (Figure 5); the spine was normal. He developed severe dysplasia of the femoral heads and necks. There was no family history, suggesting that the condition had arisen from a new dominant mutation or was inherited as a recessive trait. His radiographic findings were quite different from all the others except for patient 14, who had similar mini-epiphyses in the proximal femurs and subsequently developed severe hip dysplasia (Figure 6). She had no family history of MED, either, but her parents were consanguineous.

Prevalence of mutations in the known MED genes The causative mutation was identified here in six of the 25 patients. Taking together with the previously reported mutations, ${ }^{10,14,15,18,19}$ this means that seven mutations have now been detected in 10 of the 29 consecutive MED patients. Four had a mutation in DTDST (14\%), three in COMP (10\%) and three in MATN3 (10\%). No mutations were identified in the collagen IX genes. The known MED genes were thus responsible altogether for $34 \%$ of the cases in this cohort.

\section{Discussion}

MED is a genetically heterogeneous disorder, causative mutations having been identified in five genes in the autosomal dominant form and one gene in the autosomal recessive form. ${ }^{1-3}$ Thus far 22 COMP mutations have been reported in 27 MED probands. ${ }^{2,4-6}$ In larger studies in which multiple probands have been analysed for COMP mutations, ${ }^{4,13,21,22}$ a causative COMP mutation has been found approximately in 13\% of the probands (range $7-$ $35 \%$ ) agreeing well with the mutation frequency obtained in the current cohort (10\%). Collagen IX mutations seem to be rare causes of MED. ${ }^{2}$ MATN3 mutations are more common than collagen IX mutations, as seven mutations have been found in 10 of the 77 probands analysed (13\%). ${ }^{8-10}$ DTDST mutations have been reported in 24 MED probands, ${ }^{12-15}$ but no reliable estimates are available for their frequency in cases of MED, since most reports have been analyses of patients with features consistent with rMED (club foot, double-layered patella). In the light of previous reports, COMP and DTDST mutations seem to be the most common causes of MED.

The frequency of mutations in the known MED genes varies from one study to another, probably because of selection bias. To address this issue, we considered consecutive MED patients monitored at a single paediatric orthopaedic clinic. Altogether, seven mutations were

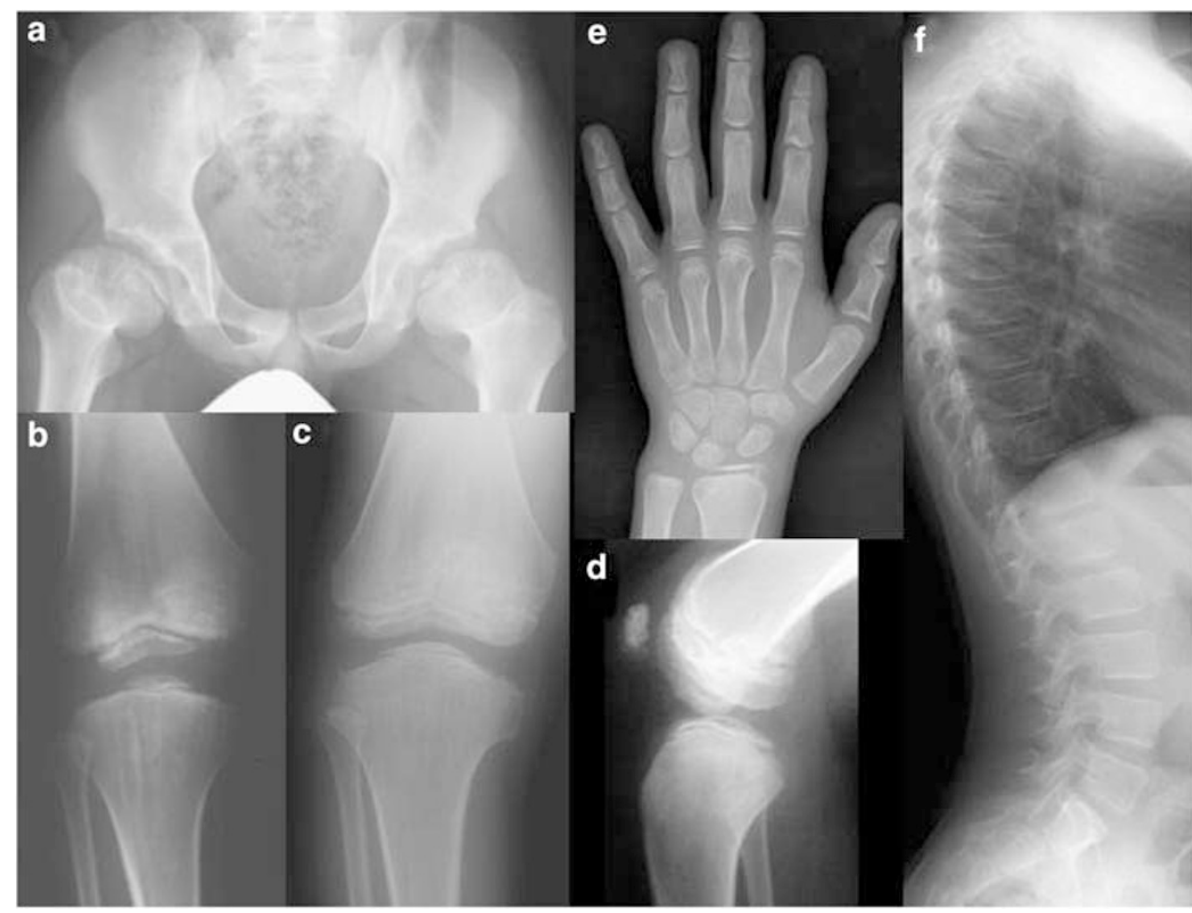

Figure 5 Patient 16 was diagnosed with MED in early childhood. At 10 years he was asymptomatic but had genua vara, increased lumbar lordosis, short stature $(<3 \%)$ and generalized joint laxity. His radiographs at age $7-10$ years showed abnormal proximal femurs with mini-epiphyses and wide, deformed femoral necks ((a) at 10 years), and mini-epiphyses at the knees ((b) at 7 years and (c) at 10 years) and hand ((e) at 7 years). Lateral view of the knee ((d) at 10 yrs) showed small and irregular patella. Lateral view of the spine $((\mathbf{f})$ at 7 years) showed increased lumbar lordosis but normal vertebrae. 


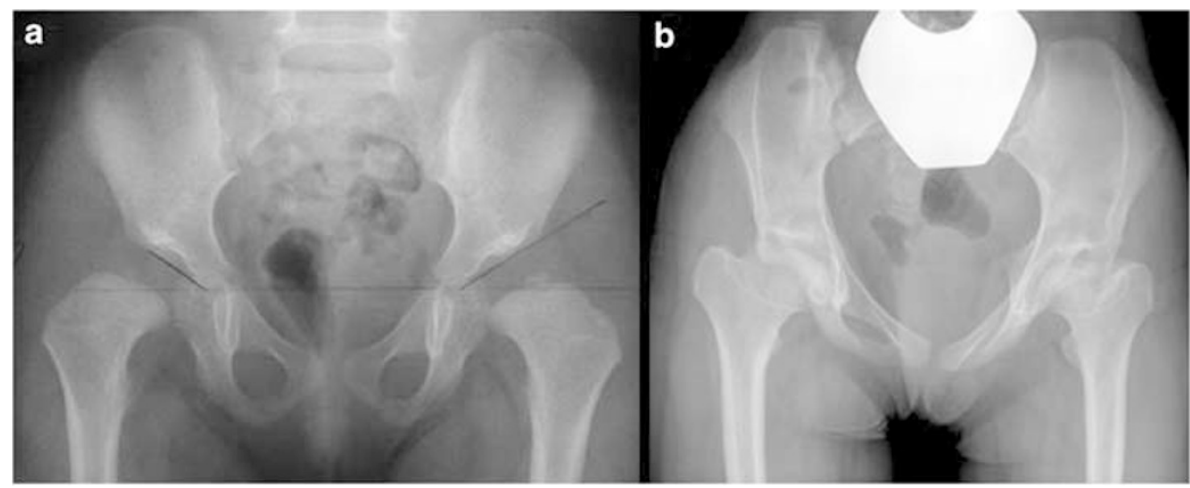

Figure 6 Patient 14 at age 3 years had similar proximal femoral mini-epiphyses and abnormal femoral necks (a) as patient 16 (see Figure 5). These resulted in severe bilateral hip deformity at age 13 years (b).

identified in 10 out of 29 patients, giving a frequency of $34 \%$ for mutations in the known MED genes. DTDST mutations were the most frequent, being found in four probands (14\%), while three probands had a COMP mutation (10\%) and three had a MATN3 mutation (10\%). Despite the relatively small sample size, the present cohort gives a representative estimate of the role of the known MED genes in the pathogenesis of this condition. The overall mutation frequency obtained here is supported by another, slightly larger study in which similar mutation frequencies were obtained. ${ }^{13}$

We failed to identify mutations in any of these genes in 19 of the 29 patients $(64 \%)$. There are several possible explanations for the low mutation frequency. The most important one is the variable phenotype of MED patients, reflecting the fact that different types of mutation in different genes are likely to result in slightly different phenotypes. It is also possible that not all mutations were detected. The number of false negatives is likely to be low, because in addition to the initial mutation screening by CSGE all candidate exons were also sequenced and RNA studies of COMP were performed in two cases. It is possible that there are rare undetected mutations in the parts of collagen IX genes or COMP not analysed or analysed only by CSGE. We have previously analysed all COL9A1, COL9A2 and COL9A3 exons in altogether 120 MED patients ${ }^{6,13,23}$ (Briggs and Ala-Kokko, (unpublished data). Since no mutations have been found by us or others in the exons not included in the present analysis and all known collagen IX mutations are splicing mutations that are located in this region, we did not extend our analysis beyond the candidate exons.

The patients with mutations in the COMP, MATN3 and DTDST genes presented with clinical and radiographic findings that are well in accordance with the phenotypes previously reported in the presence of similar mutations. Patients with MATN3 mutations present with features partially overlapping with MED caused by COMP and collagen IX mutations, ${ }^{19}$ but may be differentiated from these by a careful assessment of their skeletal radiographs. As in the other forms of MED, ${ }^{6,15}$ however, there may exist a 'window of opportunity' in mid-childhood for differentiating these forms from each other, since the radiographic findings become less characteristic towards closure of the growth plates. $\operatorname{Arg}^{718} \operatorname{Trp}^{9}$ and $\mathrm{Ala}^{735} \mathrm{Val}$ (this study) mutations in C-terminus of COMP seem to associate with myopathic symptoms which are similar to those seen in patients with a COL9A3 mutation. ${ }^{23,24}$ Some heterozygous changes in DTDST were identified in MED patients, but, since all disorders caused by DTDST mutations are recessive, $^{16}$ these changes are unlikely to affect MED phenotype; the existence of a second, unidentified mutation in a region not analysed cannot be ruled out.

Interestingly, a review of the radiographs of the patients with MED and no identified mutations revealed at least two new specific phenotypic entities. Three patients were found to have strikingly similar severe, early-onset dysplasia of the proximal femurs with almost complete absence of the secondary ossification centres and abnormal development of the femoral necks. Spinal changes, present in two patients, were significantly milder than usually seen in spondyloepiphyseal dysplasia; the vertebrae were normal in shape and the epiphyseal changes dominated. One of the patients had a family history consistent with autosomal dominant inheritance. Unfortunately, the pedigree was not large enough for a genome-wide scan to search for a gene responsible for this new MED phenotype. Another new phenotypic entity was characterized by 'mini-epiphyses', which resulted in severe dysplasia of the proximal femoral heads in two patients. Both had a normal spine, and neither had affected relatives, while the consanguinity of the parents of the latter proband suggests that this may represent another autosomal recessive form of MED.

In conclusion, the present results suggest that a significant number of patients with MED do not have mutations in any of the previously recognized MED genes. The identification of two new phenotypic entities among patients in whom mutation screening of known loci was negative supports the 
existence of additional MED loci. Future studies should aim at further delineating these phenotypes, their inheritance and their molecular pathology.

\section{Acknowledgements}

We wish to thank the patients and families who participated in the study. We thank Ms Aira Harju, Ms Satu Koljonen, Ms Minta Lumme, Mr Justin Manges and Ms Christina Troxell for their expert technical assistance and RN Catherine Barclay for her expertise in collecting the samples. This study was supported by grants from the European Commission (grant QLG1-CT-2001-02188 to L Ala-Kokko and MD Briggs), the Foundation for Paediatric Research, the Finnish Medical Association Duodecim, Finland, and an ESPE Research Fellowship sponsored by Novo Nordisk A/S (to O Mäkitie), the Canadian Institutes of Health Research and the Canadian Arthritis Network (to WG Cole), the Academy of Finland, the Arthritis Foundation, the Louisiana Gene Therapy Consortium (New Orleans, LA, USA) and HCA-The Health Care Company, Nashville, TN, USA (to L Ala-Kokko).

\section{References}

1 Unger S, Hecht JT: Pseudoachondroplasia and multiple epiphyseal dysplasia: new etiologic developments. Am J Med Genet 2001; 106: 244-250.

2 Briggs MD, Chapman KL: Pseudoachondroplasia and multiple epiphyseal dysplasia: mutation review, molecular interactions, and genotype to phenotype correlations. Hum Mutat 2002; 19: 465-478.

3 Chapman KL, Briggs MD, Mortier GR: Review: clinical variability and genetic heterogeneity in multiple epiphyseal dysplasia. Pediatr Pathol Mol Med 2003; 22: 53-75.

4 Mabuchi A, Manabe N, Haga N et al: Novel types of COMP mutations and genotype-phenotype association in pseudoachondroplasia and multiple epiphyseal dysplasia. Hum Genet 2003; 112: 84-90.

5 Song HR, Lee KS, Li QW, Koo SK, Jung SC: Identification of cartilage oligomeric matrix protein (COMP) gene mutations in patients with pseudoachondroplasia and multiple epiphyseal dysplasia. J Hum Genet 2003; 48: 222-225.

6 Jakkula E, Lohiniva J, Capone A et al: A recurrent R718W mutation in COMP results in multiple epiphyseal dysplasia with mild myopathy: clinical and pathogenetic overlap with collagen IX mutations. J Med Genet 2003; 40: 942-948.

7 Fiedler J, Stove J, Heber F, Brenner RE: Clinical phenotype and molecular diagnosis of multiple epiphyseal dysplasia with relative hip sparing during childhood (EDM2). Am J Med Genet 2002; 112: $144-153$

8 Chapman KL, Mortier GR, Chapman K, Loughlin J, Grant ME, Briggs MD: Mutations in the region encoding the von Willebrand factor A domain of matrilin-3 are associated with multiple epiphyseal dysplasia. Nat Genet 2001; 28: 393-396.

9 Mostert AK, Dijkstra PF, Jansen BR et al: Familial multiple epiphyseal dysplasia due to a matrilin-3 mutation: further delineation of the phenotype including 40 years follow-up. $\mathrm{Am}$ J Med Genet 2003; 120A: 490-497.

10 Jackson GC, Barker FS, Jakkula E et al: Missense mutations in the beta strands of the single A-domain of matrilin-3 result in multiple epiphyseal dysplasia. J Med Genet 2004; 41: 52-59.

11 Stefansson SE, Jonsson H, Ingvarsson $\mathrm{T}$ et al: Genomewide scan for hand osteoarthritis: a novel mutation in matrilin-3. Am J Hum Genet 2003; 72: 1448-1459.

12 Superti-Furga A, Neumann L, Riebel T et al: Recessively inherited multiple epiphyseal dysplasia with normal stature, club foot, and double layered patella caused by a DTDST mutation. J Med Genet 1999; 36: 621-624.

13 Czarny-Ratajczak M, Lohiniva J, Rogala $\mathrm{P}$ et al: A mutation in COL9A1 causes multiple epiphyseal dysplasia: further evidence for locus heterogeneity. Am J Hum Genet 2001; 69: 969-980.

14 Ballhausen D, Bonafe L, Terhal $\mathrm{P}$ et al: Recessive multiple epiphyseal dysplasia (rMED): phenotype delineation in eighteen homozygotes for DTDST mutation R279W. J Med Genet 2003; 40: $65-71$.

15 Mäkitie O, Savarirayan R, Bonafe L et al: Autosomal recessive multiple epiphyseal dysplasia with homozygosity for C653S in the DTDST gene: double-layer patella as a reliable sign. Am J Med Genet 2003; 122A: 187-192.

16 Rossi A, Superti-Furga A: Mutations in the diastrophic dysplasia sulfate transporter (DTDST) gene (SLC26A2): 22 novel mutations, mutation review, associated skeletal phenotypes, and diagnostic relevance. Hum Mutat 2001; 17: 159-171.

17 Unger SL, Briggs MD, Holden P et al: Multiple epiphyseal dysplasia: radiographic abnormalities correlated with genotype. Pediatr Radiol 2001; 31: 10-18.

18 Susic S, McGrory J, Ahier J, Cole WG: Multiple epiphyseal dysplasia and pseudoachondroplasia due to novel mutations in the calmodulin-like repeats of cartilage oligomeric matrix protein. Clin Genet 1997; 51: 219-224.

19 Mäkitie O, Mortier GR, Czarny-Ratajczak M et al: Clinical and radiographic findings in multiple epiphyseal dysplasia caused by MATN3 mutations: description of 12 patients. Am I Med Genet 2004; 125A: 278-284.

20 Mortier GR, Chapman K, Leroy JL, Briggs MD: Clinical and radiographic features of multiple epiphyseal dysplasia not linked to the COMP or type IX collagen genes. Eur J Hum Genet 2001; 9: 606-612.

21 Briggs MD, Hoffman SM, King LM et al: Pseudoachondroplasia and multiple epiphyseal dysplasia due to mutations in the cartilage oligomeric matrix protein gene. Nat Genet 1995; 10: 330-336.

22 Briggs MD, Mortier GR, Cole WG et al: Diverse mutations in the gene for cartilage oligomeric matrix protein in the pseudoachondroplasia-multiple epiphyseal dysplasia disease spectrum. Am J Hum Genet 1998; 62: 311-319.

23 Lohiniva J, Paassilta P, Seppanen U, Vierimaa O, Kivirikko S, AlaKokko L: Splicing mutations in the COL3 domain of collagen IX cause multiple epiphyseal dysplasia. Am J Med Genet 2000; 90: 216-222.

24 Bönnemann CG, Cox GF, Shapiro F et al: A mutation in the alpha 3 chain of type IX collagen causes autosomal dominant multiple epiphyseal dysplasia with mild myopathy. Proc Natl Acad Sci USA 2000; 97: 1212-1217.

25 Mabuchi A, Ikeda T, Fukuda A et al: Identification of sequence polymorphisms of the COMP (cartilage oligomeric matrix protein) gene and association study in osteoarthrosis of the knee and hip joints. J Hum Genet 2001; 46: 456-462.

26 Loughlin J, Mustafa Z, Dowling B et al: Finer linkage mapping of a primary hip osteoarthritis susceptibility locus on chromosome 6 . Eur I Hum Genet 2002; 10: 562-568.

27 Hästbacka J, Superti-Furga A, Wilcox WR, Rimoin DL, Cohn DH, Lander ES: Atelosteogenesis type II is caused by mutations in the diastrophic dysplasia sulphate transporter gene (DTDST): evidence for a phenotypic series involving three chondrodysplasias. Am J Hum Genet 1996; 58: 255-262.

28 Hästbacka J, de la Chapelle A, Mahtani MM et al: The diastrophic dysplasia gene encodes a novel sulfate transporter: positional cloning by fine-structure linkage disequilibrium mapping. Cell 1994; 78: 1073-1087.

Supplementary Information accompanies the paper on European Journal of Human Genetics website (http://www.nature.com/ejhg). 